\title{
Janet Tello Gilardi y Carlos Calderón Puertas (compiladores). Reglas de Brasilia: justicia para transformar la vulnerabilidad en igualdad.
}

Lima: Comisión Permanente de Acceso a la Justicia de Personas en Condición de Vulnerabilidad y Justicia en tu Comunidad/ Fondo Editorial del Poder Judicial, 2020, 332 pp.

00

Llega a mis manos una obra transformadora de la Comisión Permanente de Acceso a la Justicia de Personas en Condición de Vulnerabilidad y Justicia en tu Comunidad, del Poder Judicial de Perú. Esta comisión, un verdadero faro para la justicia latinoamericana, que activa el empoderamiento de los más frágiles de nuestra comunidad, a través de este libro y de publicaciones previas, trata de acercar al ciudadano de a pie a la justicia que, muchas veces, por circunstancias personales, le resulta esquiva. Esta nueva obra ha convocado a grandes juristas del ámbito interno e internacional. Contiene doce artículos con sendas miradas sobre diversos obstáculos en el acceso a la justicia y los caminos posibles para su superación. 


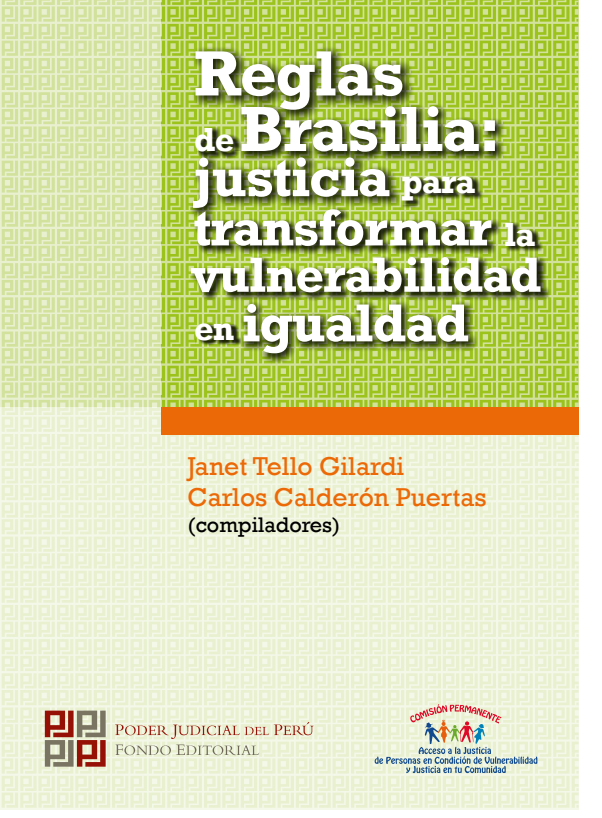

El profesor peruano Edwin Figueroa Gutarra nos enfrenta a un problema tantas veces señalado por la jurisprudencia de la Corte Interamericana: se presenta una extraterritorialidad, una sensación de exilio lingüístico cuando una parte dentro de un proceso no puede comprender el procedimiento en el cual se deciden sus derechos. Se trata casi de una expropiación del derecho propio, cuando este se enajena en vericuetos procesales y lenguajes inaccesibles para su titular. El autor no señala únicamente las dificultades lingüísticas provenientes del uso técnico del lenguaje jurídico frente a usuarios del servicio de justicia que comprenden solo el lenguaje común. Entre los asuntos a considerar aparecen la necesidad de despojar al lenguaje de ambigüedad y vaguedad; del uso del lenguaje común, sencillo y claro en las resoluciones judiciales; de evitar las expresiones discriminatorias; y de la adecuada comprensión de las actuaciones orales. Resulta evidente que para que la parte pueda empoderarse en el proceso que decide sus derechos, este debe ser asible: comprendido de modo que la parte sea dueña de su respuesta. La condición de igualdad y la satisfacción e implementación práctica de medidas que tiendan a acercar lingüísticamente los procesos para hacerlos accesibles supone parte del principio de efectividad que obliga a los estados a realizar los derechos.

El profesor español José Garberí Llobregat ofrece un análisis del tratamiento del derecho al acceso a los órganos judiciales y su evolución en la jurisprudencia del Tribunal Constitucional Español. 
Como exige la disciplina comparatista, el autor clarifica el sentido del acceso a la justicia en el derecho español como abarcador no solo del acceso a la jurisdicción, sino también del derecho a obtener una resolución judicial dictada de acuerdo con las debidas instancias recursivas. El derecho al acceso a la justicia se ve conculcado por interpretaciones manifiestamente erróneas, o basadas en rigorismo o formalismo, o normas que impongan obstáculos excesivos de forma irrazonable. Por otra parte, el marco institucional del proceso puede ser un obstáculo en sí mismo: son ejemplos los altos costes, la imposición de instancias previas en procesos de bajo monto $\mathrm{u}$ otras circunstancias similares. Es de sumo interés el desarrollo de la jurisprudencia en torno a la conformidad constitucional de determinados requisitos para acceder a la justicia, entre ellos, la imposición de plazos de caducidad en algunas circunstancias especiales, el requisito de acción administrativa previa o la fianza requerida en algunos casos.

La jurisprudencia ha admitido el criterio «pro actione», según el cual la interpretación de la norma de acceso debe realizarse siempre del modo más favorable al ejercicio de la acción. Entre las obligaciones judiciales de contenido negativo, derivadas de este principio, el autor desarrolla las interpretaciones rígidas, rigoristas y formalistas, entre otras. Asimismo, entre las obligaciones judiciales de contenido positivo existe la obligación de promover la subsanación de defectos e irregularidades procesales que obstarían la acción.

La profesora paraguaya Claudia Patricia Sanabria Moudelle y el profesor catalán Isaac Ravetllat Ballesté (hoy catedrático en Chile) se explayan sobre la defensa legal de los derechos de niños y adolescentes durante la pandemia. Luego de enunciar los principios rectores en la materia, se alarman por la invisibilidad de los niños en el hogar. El derecho a la ciudadanía se vio oscurecido por el encierro derivado de las medidas en pandemia. Una de las cuestiones más sensibles es el proceso de digitalización que produjo la 
pandemia y el riesgo de afectación consecuente de la imagen, la identidad y la privacidad como derechos personalísimos de los niños. Por otra parte, el derecho de las niñas y los niños de vivir en familia también se ha visto resquebrajado por el encierro: la adecuada comunicación con los padres en contextos de familias divididas, y los niños sin cuidados parentales, que esperaban ver satisfechos sus anhelos de vida familiar, han sido las principales víctimas. Se han visto afectados tanto el derecho a la educación como el derecho a la salud, este último por la falta de controles periódicos y falta de respuesta frente a los nuevos hábitos de los niños y las niñas. La educación implicó un desafío de reconversión para la satisfacción de derechos, en especial los que tienen relación con la socialización de los niños y el derecho a la autonomía. Un tema acuciante, abordado por los autores, es la atención integral a la víctima de abuso sexual y violencia. El secreto del hogar encubre muchos de los crímenes que se perpetúan a su amparo. Es urgente atender esta problemática que destruye sus futuros. De allí la enorme importancia de garantizar y allanar el acceso a la justicia especializada para este grupo de sujetos vulnerables.

La profesora chilena Rommy Álvarez Escudero escribe sobre un aspecto esencial que aqueja a muchos niños, niñas y adolescentes de especial vulnerabilidad. Se trata de un derecho poco abordado y tratado por la doctrina: la centralidad y superioridad del interés del niño debe ser impresa a todas las formas de filiación, cualquiera sea su origen: matrimonial o extramatrimonial, por reproducción asistida o biológica, sean los hijos queridos o no. Engendrar implica la responsabilidad de cuidado por ese niño, indisponible por los progenitores, aunque el controlador estatal pueda removerla cuando no se ejerce en interés del niño. En una palabra: todo niño tiene, en principio, el derecho a la coparentalidad. El artículo examina las obligaciones del Estado en materia de acceso a la justicia, en orden a garantizar este derecho. Existe un deber jurídico 
del padre a reconocer a su hijo. Su incumplimiento voluntario hace nacer un deber de reparación respecto del hijo. En virtud de la arquitectura del principio de coparentalidad, que establece la tríada padre, madre e hijo, la madre también resulta garante de la coparentalidad, de manera que debe informar al hijo quién es el padre (y este también es un deber jurídico, por lo que diversos sistemas comparados han dado lugar a la reparación por incumplimiento). Pensar en la filiación como un derecho subjetivo del hijo se transforma así en un derecho a la acción respectiva de la cual el Estado resulta garante.

El profesor peruano Manuel Bermúdez Tapia recontextualiza el acceso a la justicia en materia penal y familiar, en el marco interdisciplinario de la teoría del conflicto. Esta teoría permite humanizar al proceso, haciéndolo vecino de las circunstancias personales. Con frecuencia, los conflictos familiares derivan en un solo hecho que implica una demanda civil y otra penal. La división de procesos y la diversidad de abordajes que esta ocasiona no solo generan una fragmentación de la problemática, sino que someten al hecho generador a diversos parámetros de análisis que terminan encapsulando a la víctima en subsistemas jurídicos, alejándola de la inmediatez y concreción con que sus circunstancias deberían ser percibidas. Las Reglas de Brasilia, en este sentido, apuntan a un abordaje integral y contextualizado de la víctima, garantizándole un auténtico acceso a la justicia.

El profesor argentino Matías Bonavitta analiza la posición de jóvenes «habitados por la ruptura», en el sentido de que, a partir de la ruptura con la familia, la intervención del Estado es patogénica: lejos de restaurar lo perdido, vulnera los derechos de los niños. El artículo desarrolla, desde la formalidad de la psicología, en primer lugar, la lucha por la comprensión de la discapacidad y sus derechos; en segundo lugar, un recorrido histórico del abordaje de esta problemática en las intervenciones familiares en la provincia 
de Córdoba (Argentina); en tercer lugar, se narra la metodología empleada; y, por último, el caso concreto que narra el artículo. A través del encuentro con el protagonista de este capítulo, se advierte cómo un niño es retirado con frecuencia de su hogar para evitar una vulneración de derechos. Esa interacción del Estado con el niño, lejos de restituir derechos, puede devastar la subjetividad y el proceso de construcción identitaria y socialización. Los jóvenes habitados por la ruptura son quienes, a partir de la intervención estatal, tienen reacciones emocionales y mecanismos defensivos nacidos de estrategias de supervivencia por la vulneración de derechos. Así, el texto invita a revisar las formas de intervención iatrogénica por parte del Estado.

La profesora mexicana Alejandra Donají Núñez Escobar ofrece un artículo sobre el reconocimiento de la diversidad en el sistema de justicia. El asunto se inserta respecto de la evolución de la percepción jurídica de las restricciones a la capacidad y su relatividad. Los procesos concernientes a la capacidad, analizados a la luz del Amparo en Revisión n. ${ }^{\circ}$ 1368/2015, suponen un derecho a la «accesibilidad cognitiva»: que las personas, cuya capacidad jurídica se dirime, comprendan el significado del proceso que se lleva a cabo, que se entienda que las diferencias en la capacidad son parte de la diversidad humana, que se garanticen salvaguardias y apoyo que permitan la máxima autonomía posible para la persona, y que se procure el derecho a la vida independiente y a ser incluido en la comunidad. La autora llama a pensar el sistema de justicia en clave igualitaria y procura medidas para implementar dichos criterios en el marco de las Reglas de Brasilia.

El profesor mexicano Manuel Gustavo Ocampo Muñoa aborda la temática de la interculturalidad como pauta de interpretación, que debe ser incorporada metodológicamente a la formación judicial en México. En primer lugar, aporta un análisis acerca de la interculturalidad en el constitucionalismo mexicano: el país 
reconoce un pluralismo jurídico formal (se admiten derechos colectivos a los pueblos indígenas y se aceptan sistemas normativos internos aprobados por el orden jurídico vigente de cada entidad federativa, respetuosos de los derechos humanos), pero mantiene un monismo jurídico. El artículo presenta un profuso desarrollo de circunstancias en que estas reglas se aplican en los diferentes estados federativos. Si bien se ejemplifican casos de incorporación de elementos relacionados con la interculturalidad y el pluralismo jurídico para que las resoluciones judiciales adquieran sensibilidad a la pertenencia cultural, se hace indispensable avanzar en el desafío de incorporar a las currículas una transformación más profunda de las instituciones.

El investigador peruano Manuel Martín Brañas retoma la temática indígena para tratar los retrocesos y avances en la calidad de vida de las comunidades indígenas en la Amazonía peruana. Se manifiesta una preocupación justificada sobre el futuro inmediato de las comunidades indígenas. La globalización amenaza la identidad de las comunidades. Incluso, las políticas públicas que procuraron dar identidad y representación a las comunidades en el Estado peruano han desencadenado nolens volens un proceso de transformación que acaba insertando a la cultura indígena en moldes occidentales. Los jóvenes indígenas son los más castigados, pues no son conscientes de su identidad, que resulta percibida como una entelequia que encierra marginación. En este sentido, lo que se proponía como un avance tal vez sea un retroceso. Se hace imprescindible revisar las formas en que el Estado hace lugar a las comunidades indígenas para que partan de la reivindicación de su identidad y sus modos.

La funcionaria mexicana Jessica Calderón García analiza la propiedad intelectual colectiva de los pueblos indígenas. Varios instrumentos internacionales han propuesto una protección específica sobre sus derechos. De los cinco países que tienen un porcentaje 
mayor de población indígena (Bolivia, Guatemala, Perú, México y Panamá), solo Bolivia reconoce el derecho a la propiedad intelectual indígena en su Constitución federal, mientras que Guatemala, Perú, México y Panamá lo hacen en su ordenamiento jurídico interno. Luego de un profundo análisis del reconocimiento de ese derecho en los diferentes estados federativos de México, la autora concluye que se mantiene un reconocimiento deficiente y recomienda adoptar medidas afirmativas para garantizar derechos individuales y colectivos, la implementación de un registro nacional internacional para evitar el plagio, la apropiación de terceros de elementos del patrimonio intelectual y que los organismos jurisdiccionales puedan dotar de reparación integral a cualquier infracción a la tutela de la propiedad intelectual colectiva.

El profesor colombiano Diego Araque estudia la regulación de la diversidad cultural en el Código Penal peruano, a través de la naturaleza jurídica del error de comprensión de delito culturalmente condicionado. Para el autor, se trata de un error de prohibición que recae, entonces, sobre la ilicitud de la conducta o el comportamiento. El Código peruano adopta, según su interpretación, una teoría de la culpabilidad limitada: si el error es invencible, se excluye la responsabilidad; y si el error fue vencible, se atenúa la pena. El autor analiza, a partir de doctrina diversa, la naturaleza jurídica del error de prohibición cultural, sus diferencias con la inimputabilidad, cómo debe ser comprendida y en qué circunstancias corresponde su aplicación.

Los profesores malagueños Mario Millán-Franco, Laura Domínguez de la Rosa y Sofía Louise Martínez Martínez estudian el rol del trabajador social en el peritaje social de los colectivos vulnerables en la administración judicial española. Luego de explicar el sistema español y el rol que corresponde al trabajador social, advierten sobre la importancia de su contribución al proceso, puesto que se trata de un medio de prueba y no exclusivamente de 
apoyo. Si bien es el magistrado quien en última instancia le otorga valor probatorio al peritaje, la contribución de aquel a la justicia, en cuanto a lo que documenta a través del diagnóstico social, si la vulneración de un derecho humano o la configuración de una situación de riesgo para colectivos vulnerables, resulta relevante. No obstante, se advierte un desarrollo insuficiente de la metodología de trabajo y el avance en la literatura académica. En un proceso progresivamente interdisciplinario, la valoración e inserción del trabajador social es indispensable.

La obra es plurifacética, sin embargo, demuestra un hilo conductor único que coincide con los fines del trabajo de la comisión: crear una cultura jurídica transformadora que transforme vulnerabilidad en igualdad, por el camino de fortalecer resiliencias comunitarias. Celebramos la aparición del trabajo de la Comisión Permanente de Acceso a la Justicia de Personas en Condición de Vulnerabilidad y Justicia en tu Comunidad y los aportes que cada una de las voces reseñadas harán fructificar en cada lector. ¡Por muchos volúmenes más!

Ursula Basset

Centro de Investigaciones de Derecho de Familia UCA (Buenos Aires, Argentina)

Contacto: ubasset@austral.edu.ar https://orcid.org/0000-0002-2599-2259 